\title{
Routine application of the nitroblue tetrazolium test in the clinical laboratory
}

\author{
A. M. GORDON, R. M. ROWAN, T. BROWN, AND H. G. CARSON \\ From the Departments of Bacteriology and Haematology, Belvidere Hospital, Glasgow
}

SYNOPSIS A modified nitroblue tetrazolium test (NBT) is described which is suitable for routine application in the haematology or bacteriology laboratory and which provides a rapid aid to the diagnosis of bacterial infection.

Hitherto published methods have recommended the use of heparinized whole blood samples for the performance of the NBT test. However, we have demonstrated that the use of the sucrose polymer Ficoll permits the test to be carried out on buffy coats prepared from venous blood anticoagulated in sequestrene (EDTA).

The mean percentage of NBT-positive neutrophils in 60 healthy control subjects was $6 \cdot 1$. The mean percentage of NBT-positive neutrophils in 56 patients with confirmed or presumed bacterial infection was $34 \cdot 2$. Scores within the normal range were observed in 13 patients in this group. Forty-eight individuals with viral infection yielded a mean percentage of NBT-positive neutrophils of 8.5. Six patients in this group gave scores above the upper limit of normal.

Both pathological groups are described in detail and applications of this test are suggested.

Baehner and Nathan (1967) showed that while a small proportion of the neutrophil polymorphs of normal subjects could in vitro reduce the soluble dye nitroblue tetrazolium (NBT) to an insoluble formazan precipitate, leucocytes from subjects with chronic granulomatous disease were unable to effect this reaction, thus providing a sensitive diagnostic test for the latter condition. An increased spontaneous reduction of NBT to formazan by intact peripheral blood neutrophils in vitro was described by Park, Fikrig, and Smithwick (1968) as a rapid aid to the diagnosis of bacterial infection in children. Enhanced NBT reduction has been shown to be characteristic of patients with systemic bacterial infections (Feigin, Shackelford, Choi, Flake, Franklin, and Eisenberg, 1971), malaria (Andersen, 1971; Chretian and Garagusi, 1971), systemic mycoses (Park et al, 1968), and of the newborn infant (Humbert, Kurtz, and Hathaway, 1970), provided that phagocytosis in the host is operating normally at the time of testing. The absolute number and percentage of NBT-positive neutrophils is low in normal subjects, in uncomplicated viral infection, and in patients with pyrexia and leucocytosis of non-bacterial origin.

The mechanism of action of this test remains

Received for publication 26 October 1972. unclear. Since the intact membrane is not permeable to the dye (Park, 1971), alteration to the cell membrane seems necessary. This change may be induced either by phagocytosis of bacteria or liberation of their products (Park, 1971). It appears that the mechanism of reduction of NBT is dependent on the activity of NADPH oxidase within the neutrophil, since it has been shown by Baehner, Nathan, and Karnovsky (1970) that this enzyme is absent in chronic granulomatous disease, a situation in which dye reduction does not occur.

It has been suggested that the routine use of this test in the clinical laboratory may be valuable in supporting a diagnosis of systemic bacterial infection and may serve to exclude a diagnosis of viral or non-infectious disease (Park et al, 1968; Matula and Paterson, 1971). We have used a modification of the NBT technique to study patients with a variety of infective processes. The purpose of this paper is to report our experience of the test and review its general usefulness.

\section{Methods and Materials}

Two stock buffers were prepared comprising $0 \cdot 2 \mathrm{M}$ solution of monobasic sodium phosphate (solution A: $31.2 \mathrm{~g} \mathrm{NaH}_{2} \mathrm{PO}_{4} .2 \mathrm{H}_{2} \mathrm{O} /$ litre) and an $0.2 \mathrm{M}$ 
solution of dibasic sodium phosphate (solution B: $28.3 \mathrm{~g} \mathrm{Na}_{2} \mathrm{HPO}_{4} /$ litre). A final working buffer was prepared by adding $100 \mathrm{ml}$ deionized water to 20 $\mathrm{ml}$ of solution A and $80 \mathrm{ml}$ of solution $\mathrm{B}$. The $p \mathrm{H}$ of the final buffer was 7.21.

The dye employed was nitroblue tetrazolium, crystalline grade III (Sigma Chemical Company, St Louis, USA). The dye was dissolved in the phosphate buffer to a final concentration of $0.2 \%$. In order to evaluate the effect of sucrose polymer on the reaction two working NBT reagents were prepared, one of which contained Ficollsucrose polymer (Pharmacia, Uppsala, Sweden) in a concentration of $200 \mathrm{mg} / \mathrm{ml}$. The final $p \mathrm{H}$ of the reagents was $7 \cdot 25$. They were stored at $+4^{\circ} \mathrm{C}$ until used.

Blood was obtained from patients and controls by clean venepuncture using dry plastic syringes with $21 \mathrm{~g}$ needles. Initially, duplicate samples using EDTA and heparin as anticoagulant were compared. Subsequently, sequestrene (EDTA) only was used as the anticoagulant. Specimens were processed as soon as possible after collection but, where delay occurred, samples were refrigerated at $+4^{\circ} \mathrm{C}$.

Siliconized disposable sedimentation tubes (Seditube: Becton, Dickinson \& Co, New Jersey, USA) were filled with blood and centrifuged at $1500 \mathrm{~g}$ for eight minutes. The supernatant plasma was discarded into $10 \%$ Chloros and the buffy coats were transferred by fine Pasteur pipettes to $75 \mathrm{~mm} \times 5$ $\mathrm{mm}$ siliconized test tubes. An equal volume of the NBT reagent was added, gently mixing, and the tubes were incubated for 30 minutes in a $37^{\circ} \mathrm{C}$ water bath and then for 15 minutes at room temperature. The contents were again mixed gently before preparing films on clean glass slides. Films were air-dried, fixed by gentle heat, and then counterstained with $0.5 \%$ aqueous safranin (Gurr) for two minutes. The slides were finally washed in water for two minutes and allowed to drain dry. Counts of formazan-positive cells were performed under oil immersion at a magnification of $\times 1000$. As a routine 100 cells were counted. Cells exhibiting a discrete particulate cytoplasmic distribution of formazan in addition to those showing very dense deposits of formazan (block positivity) were regarded as positive cells.

Samples from 60 healthy volunteers and from 104 patients were processed. The control subjects comprised 30 males (age range 20-45 yr) and 30 females (age range 18-31 yr). The patients were suffering from a wide variety of clinical syndromes. A clinical diagnosis of viral infection was made in $\mathbf{4 8}$ patients, in 23 of whom the diagnosis was confirmed by the demonstration of a virus in secretions or by significant titres in serological tests. Fifty-six patients with clinical features suggestive of a bacterial infection were studied. The diagnosis was fully confirmed by the isolation of a bacterial pathogen or by significant serological results in 45 individuals. In the remaining 11 patients the diagnosis was based solely on the clinical impression. The illnesses from which the patients were suffering are set out in Tables I and II.

\begin{tabular}{lc}
\hline Clinical Diagnosis & No. of Cases \\
\hline Upper respiratory tract infection & 5 \\
Lower respiratory tract infection & 17 \\
Soft tissue infection & 9 \\
Pyogenic meningitis & 16 \\
Septicaemias & 7 \\
Brucellosis & 1 \\
Leprosy & 1 \\
\hline
\end{tabular}

Table I Diagnoses in 56 subjects with bacterial infection

\begin{tabular}{ll}
\hline Clinical Diagnosis & No. of Cases \\
\hline Acute respiratory tract infection & 19 \\
Aseptic meningitis & 14 \\
Infectious mononucleosis & 7 \\
Measles & 4 \\
Mumps & 2 \\
Vaccinia & 2 \\
\hline
\end{tabular}

Table II Diagnoses in 48 cases patients with viral infection

\section{Results}

Our preliminary experiments demonstrated that when Ficoll was incorporated in the test systems, extravasation of formazan from neutrophils did not occur. Furthermore, a comparison of EDTA and heparin as anticoagulant showed that the former resulted in superior staining (block positivity as opposed to stippling), improved cell definition, and prevented cell agglomeration. Accordingly, we have routinely employed sequestrene as the anticoagulant and have incorporated Ficoll sucrose polymer in the NBT reagent.

The Figure shows the distribution of NBT scores in the subjects studied. In the 60 healthy controls, the mean percentage of NBT-positive neutrophils was 6.1 (6.3 in the males; 5.8 in the females), the range being $1-14 \%$. A score of $14 \%$ was, therefore, taken as the upper limit of normality in this series. In the 56 subjects with confirmed or presumed bacterial infections, the mean percentage of NBT-positive neutrophils was 34.2 with a range of 2 to 75 . The NBT scores were above the upper limit of normality in 43 of the patients while in the remaining 13 the count was within the normal range (Table III). The 


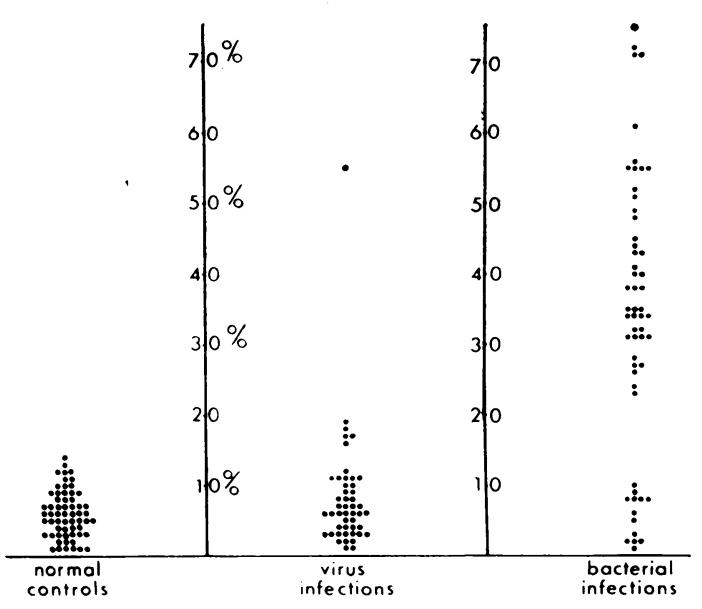

Fig. Distribution of NBT scores in control subjects and patients

mean percentage of NBT-positive neutrophils in the 48 individuals with viral illnesses was 8.5 with a range of 1-55. Forty-two of these patients had scores within the normal range while six yielded scores above the upper limit of normal (Table IV).

\begin{tabular}{ll}
\hline Diagnosis & Score (\%) \\
\hline Leprosy & 8 \\
Brucellosis & 1 \\
Primary pulmonary tuberculosis (3 cases) & $3,2,10$ \\
Meningococcal meningitis (3 cases) & $5,8,8$ \\
Meningococcal septicaemia & 2 \\
Pyogenic meningitis & 9 \\
Pseudomonas bronchopneumonia & 2 \\
Toxic epidermal necrolysis & 6 \\
Pulmonary embolism & 8 \\
\hline
\end{tabular}

Table III Low NBT scores in patients with bacterial infections

\begin{tabular}{ll}
\hline Diagnosis & Score (\%) \\
\hline M. pneumoniae infection & 17 \\
Measles encephalitis & 18 \\
Aseptic meningitis & 17 \\
Mumps meningitis (2 cases) & 19,16 \\
Mumps orchitis & 55 \\
\hline
\end{tabular}

Table IV Elevated NBT scores in patients with viral infection

\section{Discussion}

In our experience, the nitroblue tetrazolium test has proved to be simple and rapid. The test yields reliable and reproducible results.
Methods previously described (Park et al, 1968; Matula and Paterson, 1970; Freeman and King, 1971) have been based upon incubation of heparinized whole blood with the dye. Freeman and King (1972a) have stated that if the 'whole blood' method is not employed the results will be unreliable and if EDTA is used as the anticoagulant inconsistent and low results will be obtained.

Stuart and Simpson (1970) found that the inert synthetic sucrose polymer Ficoll had a protective effect on leucocytes during incubation at $37^{\circ} \mathrm{C}$, protection appearing to be due to preservation of the cytoplasmic membrane. Preliminary experiments with and without Ficoll in the NBT reagent showed that in the case of the former extravasation of formazan from the neutrophils did not occur and that cell integrity was preserved, thus suggesting that Ficoll did indeed effect membrane stabilization in the presence of EDTA.

Further experiments comparing EDTA with heparin as anticoagulant showed that while formazan staining was satisfactory with the latter, cell definition was relatively poor and clumping rendered counting difficult.

The use of buffy coat preparations did not impair the cytological quality of the preparations and facilitated counting of formazan-positive cells in the presence of neutropenia.

Our modification of the NBT test requires more blood and takes longer to perform than the technique originally described by Park et al (1968), but we are still able to obtain a result within one hour of commencement of the test. We have found that a centrifugation time of eight minutes at $1500 \mathrm{~g}$ produces the most satisfactory buffy coats while an incubation period of 30 minutes at $37^{\circ} \mathrm{C}$ followed by 15 minutes at room temperature results in optimal formazan precipitation. The choice of safranin as the counterstain permits easy identification of cell type and provides excellent contrast.

As performed in this laboratory, the NBT test is shown to possess a useful capacity to distinguish patients with active bacterial infection from those with non-bacterial inflammatory disease in both adult and childhood populations. This observation is supported by the work of Matula and Paterson (1971) and Feigin et al (1971).

Two criteria, however, must be fulfilled before the results of NBT testing become valid. These are (1) that all components of phagocytosis are operative in the host at the time of testing, and (2) that infection involves the systemic circulation (Park, 1971). It is known that an elevated NBT response in bacterial infection will return to normal within one to 14 days of starting antibiotic therapy and in fact may return to normal before clinical defervescence of 
infection (Matula and Paterson, 1970). This test may demonstrate the efficacy of antibiotic therapy in a particular individual. However, at the same time, it is important to realize that prior chemotherapy limits the validity of the test as a diagnostic tool. It is, therefore, important to withdraw diagnostic samples before initiating antibiotic therapy.

False positive and false negative reactions are not uncommon (Lancet, 1971). Two subjects from our proposed control group were excluded on the basis of enhanced NBT reduction one day after typhoid/ paratyphoid vaccination. Elevated responses have been found in Mycoplasma pneumoniae infection (Freeman and King, 1972b). One patient in our series with serologically confirmed Mycoplasma pneumoniae infection showed a marginal increase. Elgefors and Olling (1972) have reported high NBT values in patients with proven viral meningitis, including mumps complicated by orchitis, pancreatitis, or encephalitis. In our series elevated NBT scores were recorded in three patients with mumps. Two were complicated by aseptic meningitis and showed marginal elevation of the NBT score. In addition we observed slight increases in single cases of measles encephalitis and aseptic meningitis.

False negative results of the NBT test have been recorded when host phagocytic systems have not been functioning normally as in chronic granulomatous disease of childhood (Baehner and Nathan, 1968). Furthermore, localized infection, not involving the systemic circulation, will also give rise to a low result. We have investigated a patient with toxic epidermal necrolysis, a staphylococcal infection characterized by intraepidermal cleavage, in whom a normal score was recorded. It is worth emphasizing that initially localized bacterial infections may become systemic with a resultant increase in NBT response.

Matula and Paterson (1971) reported negative NBT responses in active mycobacterial infection. Park et al (1968) found normal NBT values in patients with pulmonary tuberculois but subsequently recorded elevated values in patients with miliary tuberculosis or tuberculous meningitis (Park, 1971). Our experience is in overall agreement with these findings. We studied seven patients with active mycobacterial disease. One patient with leprosy and three patients with primary pulmonary tuberculosis gave NBT scores well within the limits of normality, but one child with proven tuberculous meningitis and three adults with active pulmonary tuberculosis complicated by bronchiectasis gave elevated scores. We have examined one case of brucellosis which yielded a low score, yet another example of diminished dye reduction in granulomatous infection.
Positive NBT tests have been recorded in patients with bacterial meningitis (Park et al, 1968; Matula and Paterson, 1971). However, in agreement with the findings of Esposito and De Lalla (1972), who reported negative responses in some cases of bacterial meningitis, we have obtained very low NBT values in four patients with early meningococcal infection one of whom had a meningococcal septicaemia. We were able to excludea defect of phagocyte function in these cases by the demonstration of very high NBT positivity in the neutrophils in the cerebrospinal fluid, while none of the patients had apparently received antibiotics before admission to hospital. We encountered two further patients with clinical features suggestive of systemic bacterial infection in whom, however, normal NBT values were obtained. One patient who was receiving a course of radiotherapy for Hodgkin's disease developed a descending Pseudomonas bronchopneumonia. An NBT test performed at the time of maximal upset gave a score of only $2 \%$. Further study on the effect of irradiation on NBT reduction is currently being undertaken. A second patient with high fever, marked leucocytosis, and with clinical and radiological features suggestive of widespread bronchopneumonia showed only $8 \%$ NBT-positive neutrophils. This patient was subsequently shown to have suffered a major pulmonary thromboembolic episode and was submitted to embolectomy. We suggest that the NBT test may be of value in the differential diagnosis of such pulmonary syndromes.

There are several applications of the NBT test. First, this test is of value in the investigation of patients with defects in phagocytic function, particularly chronic granulomatous disease in which it provides a sensitive screening test especially when the stimulated version is employed. Secondly, the NBT test serves as an aid in differentiating patients with bacterial infection from those with non-bacterial illness in whom the clinical features mimic bacterial infection. Low NBT scores are found in viral or non-infectious disease with the exception of the false-negative results previously itemized. Thirdly, the test may be useful in monitoring patients at high risk of infection and in the detection of early bacterial infection in such individuals. This group includes patients who are immunosuppressed for any reason, patients on chronic renal dialysis programmes, or in intensive care units, and individuals with indwelling cannulae and catheters. Finally, this test may provide useful information when differentiating between pulmonary thromboembolism and pneumonia.

We wish to thank Dr R. G. Sommerville for access to virological data and the staff of the Department 
of Infectious Diseases at Belvidere Hospital for assistance in the collection of specimens.

\section{References}

Andersen, B. R. (1971). NBT test in malaria. (Letter). Lancet, 2, 317. Baehner, R. L., and Nathan, D. G. (1967). Leucocyte oxidase: defective activity in chronic granulomatous disease. Science, $155,835-836$.

Baehner, R. L., and Nathan, D. G. (1968). Quantitative nitroblue tetrazolium test in chronic granulomatous disease. New Engl.J. Med., 278, 971-976.

Baehner, R. L., Nathan, D. G., and Karnovsky, M. L. (1970). Correction of metabolic deficiencies in the leucocytes of patients with chronic granulomatous disease. J. clin. Invest., 49, 865-870.

Chretian, J. H., and Garagusi, V. F. (1971). NBT test in parasitic disease. (Letter). Lancet, 2, 549.

Elgefors, B., and Olling, S. (1972). NBT test in viral meningitis. (Letter). Lancet, $1,967$.

Esposito, R., and De Lalla, E. (1972). NBT test in bacterial meningitis. (Letter). Lancet, 1, 747-748.

Feigin, R. D., Shackelford, P. G., Choi, S. C., Flake, K. K., Franklin, F. A., Jr., and Eisenberg, C. S. (1971). Nitroblue tetrazolium dye test as an aid in the differential diagnosis of febrile disorders. J. Pediat., 78, 230-237.

Freeman, R., and King, B. (1971). A modification to the NBT test. (Letter). Lancet, 2, 1154.

Freeman, R., and King, B. (1972a). Nitroblue tetrazolium test and neutropenia. (Letter). Lancet, 1, 533-534.

Freeman, R., and King, B. (1972b). NBT test and mycoplasma. (Letter). Lancet, 1, 962.

Humbert, J. R., Kurtz, M. L., and Hathaway, W. E. (1970). Increased reduction of nitroblue tetrazolium by neutrophils of newborn infants. Pediatrics, 45, 125-128.
Lancet (1971). Leading article. Nitroblue tetrazolium: a routine test? Lancet, 2, 909-910.

Matula, G., and Paterson, P. Y. (1970). Spontaneous in vitro nitroblue tetrazolium reduction: a discriminatory test for bacterial infection in adults. $J$. clin. Invest., 49, 62a.

Matula, G., and Paterson, P. Y.(1971). Spontaneous in vitro reduction of nitroblue tetrazolium by neutrophils of adult patients with bacterial infection. New. Engl. J. Med., 285, 311-317.

Park, B. H. (1971). The use and limitations of the nitroblue tetrazolium test as a diagnostic aid. J. Pediat., 78, 376-378.

Park, B. H., Fikrig, S. M., and Smithwick, E. M. (1968). Infection and nitroblue tetrazolium reduction by neutrophils; a diagnostic aid. Lancet, 2, 532-534.

Stuart, J., and Simpson, J. S. (1970). Dehydrogenase enzyme cytochemistry of unfixed leucocytes. J. clin. Path., 23, 517-521.

\section{Addendum}

Since the completion of this manuscript, Wollman and his colleagues (1972) have reported that the NBT test is useful in monitoring the occurrence of bacterial infections in uraemic patients and have suggested that the test may be of value in the differentiation between infection and rejection in the renal transplant patient.

\section{Reference}

Wollman, M. R., Brennan, B. L., Stenzel, K. H., David, D. S., Lewy, J. E., Ruben, A. L., and Miller, D. R. (1972). The Nitroblue Tetrazolium test. Lancet, 2, 289-292. 